\title{
Activation of lymphocytes and increased interleukin-5 levels in bronchoalveolar lavage fluid in acute eosinophilic pneumonia
}

\author{
H. Taniguchi*, J-i. Kadota ${ }^{+}$, T. Fujii ${ }^{+}$, Y-i. Matsubara ${ }^{+}$, S. Katoh*, H. Mukae*, S. Matsukura*, \\ S. Kohno
}

Activation of lymphocytes and increased interleukin-5 levels in bronchoalveolar lavage fluid in acute eosinophilic pneumonia. H. Taniguchi, J-i. Kadota, T. Fujii, Y-i. Matsubara, S. Katoh, H. Mukae, S. Matsukura, S. Kohno. C) ERS Journals Ltd 1999.

ABSTRACT: Acute eosinophilic pneumonia (AEP) is a recently described illness and the number of case reports has increased during the last few years. However, the role of interleukin (IL)-5 and activated lymphocytes in the pathogenesis or activity of AEP is still not clear

The clinical features, lymphocyte surface analysis and IL-5 concentrations in bronchoalveolar lavage fluid (BALF) and peripheral blood (PB) of a young female patient with AEP are described before and at 2 weeks, 4 weeks and 6 months after a 3day course of $i . v$. methylprednisolone.

Serum and BALF concentrations of IL-5 before treatment were 5,200 and 8,400 $\mathrm{pg} \cdot \mathrm{mL}^{-1}$, respectively. Activated CD4 lymphocytes bearing CD25 and human leukocyte antigen (HLA)-DR in BALF were higher than in PB. Treatment caused a rapid fall in these cells and levels of IL-5 in BALF returned to normal levels in parallel with clinical improvement. There was no evidence of recurrence after cessation of steroid therapy. In contrast, eosinophilia in BALF persisted for 4 weeks after steroid therapy in spite of normalization of the chest radiograph and arterial blood gases. The number of CD8+CD11b- (suppressor/cytotoxic) T-cells subsequently increased while the number of CD8+CD11b+ cells decreased.

These results suggest that activated CD4 cells and interleukin-5 elevation contribute to the development of acute eosinophilic pneumonia rather than persistent eosinophilia in the lung and that a short course of steroid therapy may effectively control acute eosinophilic pneumonia.

Eur Respir J 1999; 13: 217-220.

Acute eosinophilic pneumonia (AEP) is characterized by a rapid onset of clinical symptoms and signs, associated with the presence of diffuse pulmonary infiltrates on the chest radiograph and a high number of eosinophils in the alveoli $[1,2]$. The number of reports describing cases with AEP have recently increased and several of these have demonstrated elevation of interleukin (IL)-5 levels in serum and bronchoalveolar lavage fluid (BALF) [3-6]. IL5 is very important among eosinophil-related cytokines because of its selective function of eosinophil activation and recruitment $[7,8]$, and is produced mainly by T-helper lymphocytes. However, only a few reports have described the role of IL-5 in the pathogenesis or disease activity of AEP [5, 9]. Furthermore, the relationship between eosinophil accumulation in the lung and IL-5 is still obscure in this disease.

In this study, we attempted to elucidate the relationship between eosinophilia, activated lymphocytes and IL-5 on the one hand and disease activity of AEP on the other. Serial analysis of surface markers of lymphocytes in BALF and peripheral blood (PB) and serial measurements of BALF and serum IL-5 concentrations were performed before and after steroid therapy.
${ }^{+}$The Second Dept of Internal Medicine, Nagasaki University School of Medicine, Nagasaki; and *The Third Dept of Internal Medicine, Miyazaki Medical College, Miyazaki, Japan

Correspondence: H. Taniguchi The Third Dept of Internal Medicine Miyazaki Medical College 5200 Kihara, Kiyotake, Miyazaki, 889-1692 Japan

Fax: 81985857591

Keywords: Acute eosinophilic pneumonia bronchoalveolar lavage fluid interleukin-5

lymphocyte activation

Received: February 101998

Accepted after revision September 71998

\section{Case report}

A 21-yr-old Japanese female was admitted to Nagasaki University Hospital with a 2-day history of dry cough, fever and exertional dyspnoea. She was treated with cefatrizine and mefenamic acid one day before admission following a visit to the family physician. She was a college student and began to smoke 1 week before the onset of symptoms. She had a history of atopic dermatitis. She had taken no medication prior to the illness and there was no evidence of parasitic infections.

On admission, she was febrile with a body temperature of $39.6^{\circ} \mathrm{C}$. Physical examination of the chest showed coarse crackles bilaterally at lower lung fields but no cyanosis was observed. No other abnormalities were present. The peripheral white cell count was $13,000 \cdot \mathrm{mm}^{-3}$, with a differential count of $15 \%$ band cells, $70 \%$ neutrophils, $8 \%$ lymphocytes, $3 \%$ monocytes, and $4 \%$ eosinophils. Arterial blood gases on breathing room air were arterial carbon dioxide tension $\left(\mathrm{Pa}_{\mathrm{a}} \mathrm{CO}_{2}\right) 5.0 \mathrm{kPa}(38 \mathrm{mmHg})$, arterial oxygen tension $\left(\mathrm{Pa}_{\mathrm{a}} \mathrm{O}_{2}\right) 6.2 \mathrm{kPa}(47 \mathrm{mmHg}), \mathrm{pH} 7.43$, and $\mathrm{HCO}_{3}{ }^{-} 25.4 \mathrm{mEq} \cdot \mathrm{L}^{-1}$. The chest radiograph and computed tomography scan showed diffuse ground glass appearance, 
Kerley B lines, multiple patchy infiltrates, and a small pleural effusion (fig. 1). Gram and acid-fast stains of the sputum were negative. Bronchoscopy performed on the day of admission showed normal endobronchial anatomy. BALF obtained from the right middle lobe was cloudy and yellowish in colour, and contained a high number of cells $\left(3.5 \times 10^{5}\right.$ cells $\left.\cdot \mathrm{mL}^{-1}\right)$. BALF differential cell count showed $13 \%$ macrophages, $22 \%$ lymphocytes, $1 \%$ neutrophils and $64 \%$ eosinophils. Staining of BALF with Gram and acid-fast stain did not show any pathogenic agents. Cultures of BALF yielded no fungi, bacteria or acid-fast bacillus growth. A diagnosis of AEP was made based on clinical course and BAL findings although transbronchial lung biopsy was not performed because of the severe hypoxaemia.

Intravenous methylprednisolone therapy $(1,000 \mathrm{mg}$. day $^{-1}$ ) commenced immediately after bronchoscopy and continued for 3 days. This resulted in a rapid resolution of symptoms and normalization of the chest radiograph within 3 days. On the seventh day after treatment began, the
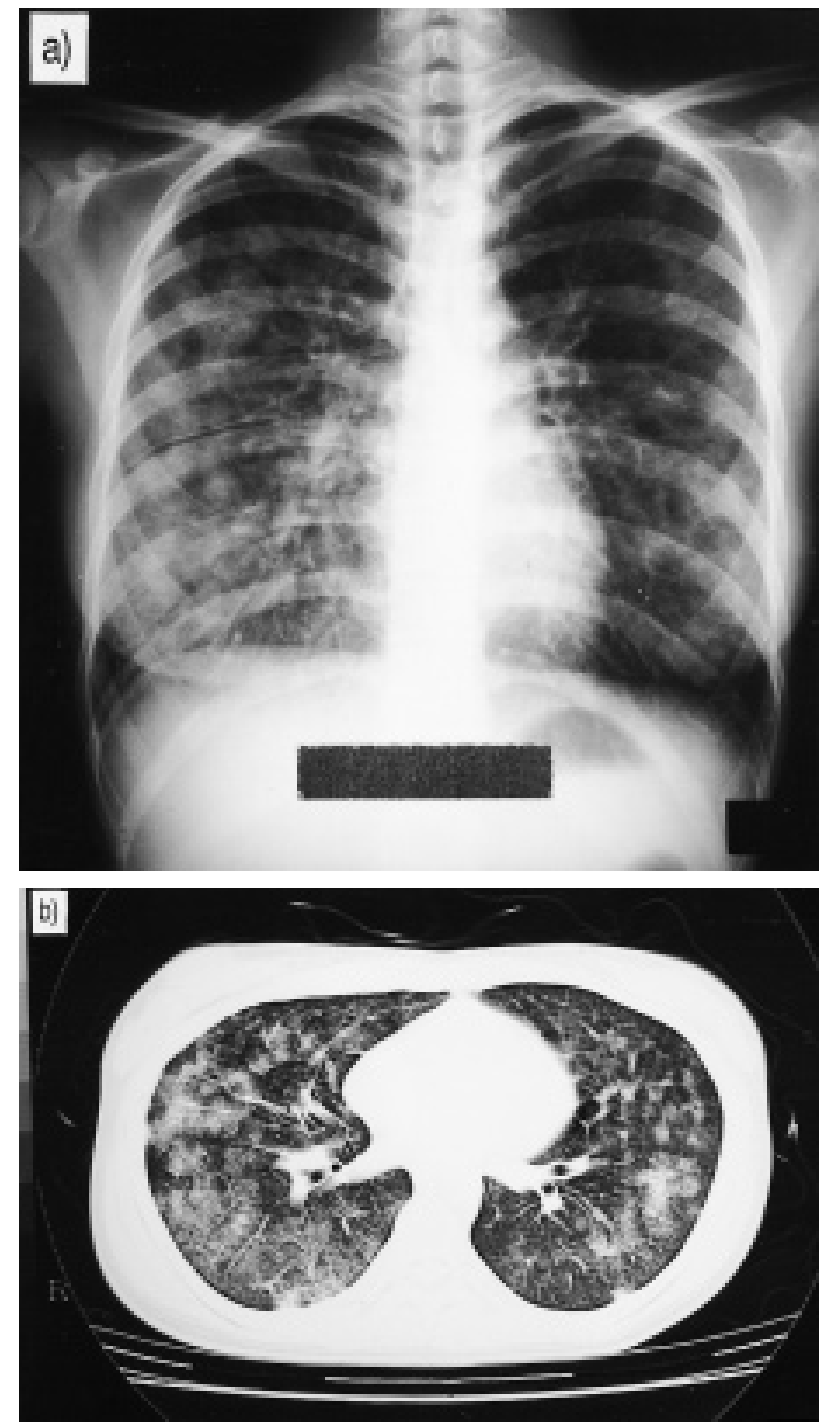

Fig. 1. - a) Chest radiograph; and b) chest computed tomography on the day of admission. Note the presence of diffuse bilateral infiltrate shadows. white blood cell count decreased to 11,000 cell $\cdot \mathrm{mm}^{-3}$ with $31 \%$ eosinophils. Resting, room air arterial blood gas analysis showed improvement of $\mathrm{Pa}, \mathrm{O}_{2}$ to $13.8 \mathrm{kPa}$ (104 $\mathrm{mmHg}$ ) within 8 days after treatment. On the 13th day after treatment began, the white blood cell count and eosinophils returned to normal levels. However, eosinophilia was still present in BALF at least 4 weeks after steroid therapy in spite of normalization of the chest radiograph and arterial blood gases. Six months after the onset of symptoms, the number of white blood cells and differential count in BALF were within normal limits (table 1). The patient showed no recurrence of symptoms after treatment.

The concentrations of IL- 5 in serum and BALF on the day of admission, measured using a sandwich enzymelinked immunosorbent assay (ELISA), were 5,200 and $8,400 \mathrm{pg} \cdot \mathrm{mL}^{-1}$, respectively. Two weeks after steroid therapy, both serum and BALF concentrations of IL-5 were reduced to $<20 \mathrm{pg} \cdot \mathrm{mL}^{-1}$. These levels remained persis-tently $<20 \mathrm{pg} \cdot \mathrm{mL}^{-1}$ at 4 weeks and 6 months follow-up.

Several lymphocyte surface markers were also examined for in PB and BALF before and after treatment by the method described below. Whole blood was placed into tubes containing ethylenediaminetetraacetic acid. Erythrocytes in PB were lysed with distilled water, and the remaining cells and BALF cells were washed once and resuspended in cold phosphate-buffered saline (PBS) containing 3\% foetal calf serum (FCS) and $0.05 \%$ sodium azide. A total of $10 \mu \mathrm{L}$ of each monoclonal antibody: fluorescein isothiocyanate (FITC)-conjugated monoclonal antibody against CD4, CD8, human leukocyte antigen (HLA)-DR and CD25 (Becton Dickinson, Mountain View, $\mathrm{CA}$, USA); and phycoerythrn (PE)-conjugated monoclonal antibody against CD4, CD8, CD45RO and CD11b (Becton Dickinson) were added to $100 \mu \mathrm{L}$ of resuspended $\mathrm{PB}$ and BAL pellets. The cells were incubated for $15 \mathrm{~min}$ on ice and then washed once in cold PBS. The cells were kept in the dark at $4^{\circ} \mathrm{C}$ until analysis. Two-colour immunofluorescence analysis of PB and BALF lymphocytes was performed by flow cytometry (FACS Calibur; Becton Dickinson). Lymphocytes were gated by forward and sidelight scattering.

Before treatment, the percentages of $\mathrm{CD} 4+, \mathrm{CD} 4+$ HLA-DR,+ CD4+CD25+, CD4+CD45RO+ cells and CD4 to $\mathrm{CD} 8$ ratio in BALF were higher than PB (tables 1 and fig. 2). In contrast, no differences were observed in the percentages of CD8+HLA-DR+ and CD8+CD25+ lymphocytes between BALF and PB (fig. 2). The CD4 to CD8 ratio decreased 2 weeks after treatment (table 1 ). The

Table 1. - Serial cytological examinations of bronchoalveolar lavage fluid before and after therapy

\begin{tabular}{lcccc}
\hline & & \multicolumn{3}{c}{ After therapy } \\
\cline { 3 - 5 } & Before & & & \\
& therapy & 2 weeks & 4 weeks & 6 months \\
\hline Total cell count & 3.5 & 3.43 & 3.68 & 1.69 \\
$\quad \times 10^{5}$ cells·mL & -1 & & & \\
Macrophages \% & 13.5 & 31.9 & 42.4 & 86.7 \\
Lymphocytes \% & 22.0 & 11.2 & 22.2 & 11.1 \\
Neutrophils \% & 1.0 & 0.9 & 0 & 1.2 \\
Eosinophils \% & 63.5 & 56.0 & 35.4 & 1.0 \\
CD4/CD8 ratio & 4.39 & 0.77 & 0.53 & 0.49 \\
\hline
\end{tabular}




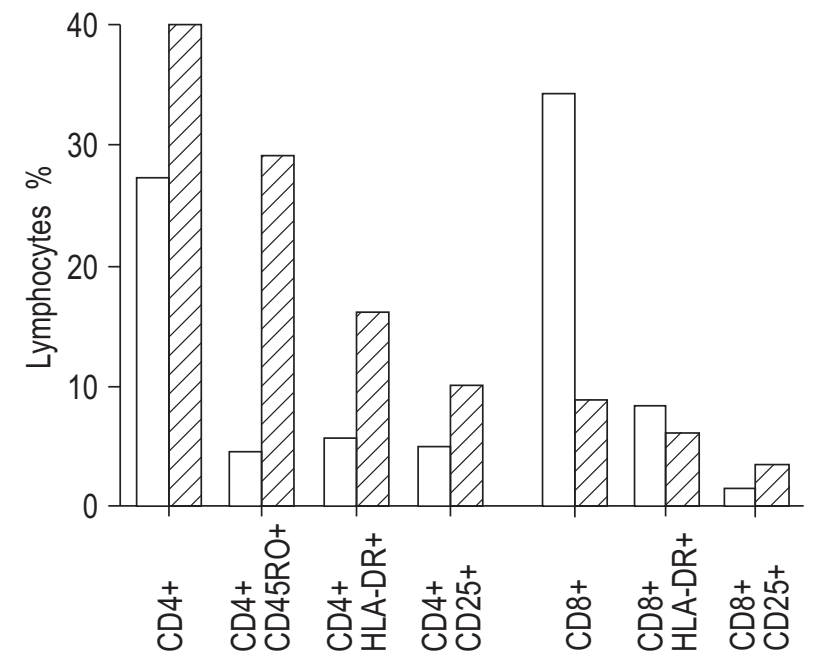

Fig. 2. - The percentage of different types of lymphocytes in the peripheral blood $(\square)$ and bronchoalveolar lavage fluid (ש) before treatment. HLA: human leukocyte antigen.

number of lymphocytes in BALF during the follow-up period is shown in figure 3 . The number of activated CD4+ lymphocytes decreased immediately after therapy. In contrast, the numbers of CD8+CD11b- and CD8+CD11b+ cells were not elevated before therapy and both increased 2 weeks after therapy. The number of CD8+CD11b- cells subsequently increased, while the number of $\mathrm{CD} 8+$ $\mathrm{CD} 11 \mathrm{~b}+$ cells decreased 4 weeks after therapy.

\section{Discussion}

In this case, the concentration of IL-5 in BALF was high compared with that in serum. These data agree with those of two AEP cases reported by ALLEN et al. [9], and suggest that IL-5 was probably produced in the lung. Elevated IL-5 in the lung may initiate the recruitment of eosinophils, increase eosinophil survival, and enhance the release of mediators from eosinophils, which in turn increases the permeability of blood vessels $[7,8]$. Since IL-5 is produced mainly by T-helper lymphocytes, we also analysed the expression of the activation antigen CD25 and HLA-DR of T-cells. Expression of CD25 is a marker of recent activation of T-cells since CD25 appears within hours after initial stimulation, is transient and disappears in the absence of continuous stimulation [10]. Furthermore, these cells could be the main cytokine-producing cells in various lung diseases, such as allergic and non-allergic asthma, eosinophilic pneumonia and allergic bronchopulmonary aspergillosis [11]. The major finding in this case report was that CD25 and HLA-DR bearing CD4+ T-cells were increased in the lung but not in $\mathrm{PB}$, indicating that CD4 cells were activated in the lung by a local immune response. In contrast, the percentage of CD8+ T-cells was not increased and these cells were not activated in either BALF or PB. The high number of recently activated CD4 cells suggests active production of IL- 5 by these cells in the lungs of this patient. Using an experimental mouse model, OKUDAIRA et al. [12] reported that T-cells are essential and T-cell-derived IL-5 is important for accumulation of eosinophils in the lung. Considered together, these findings

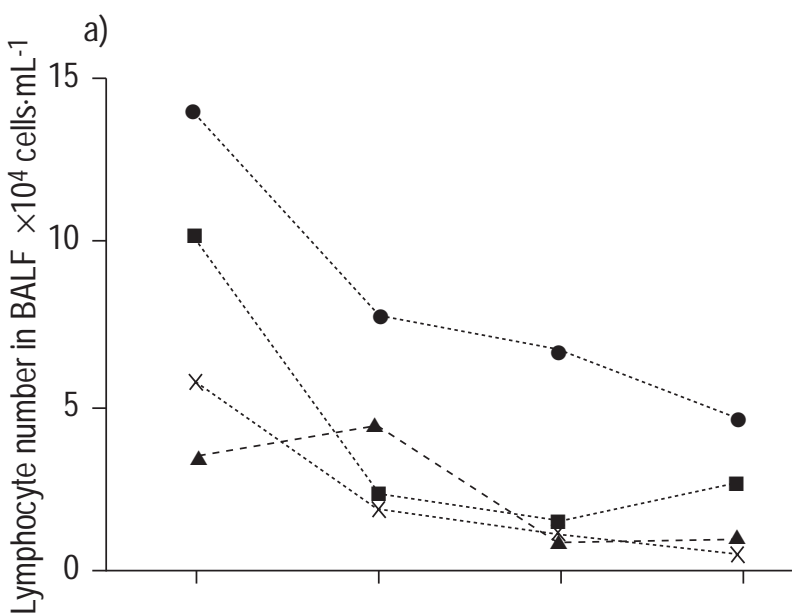

b)

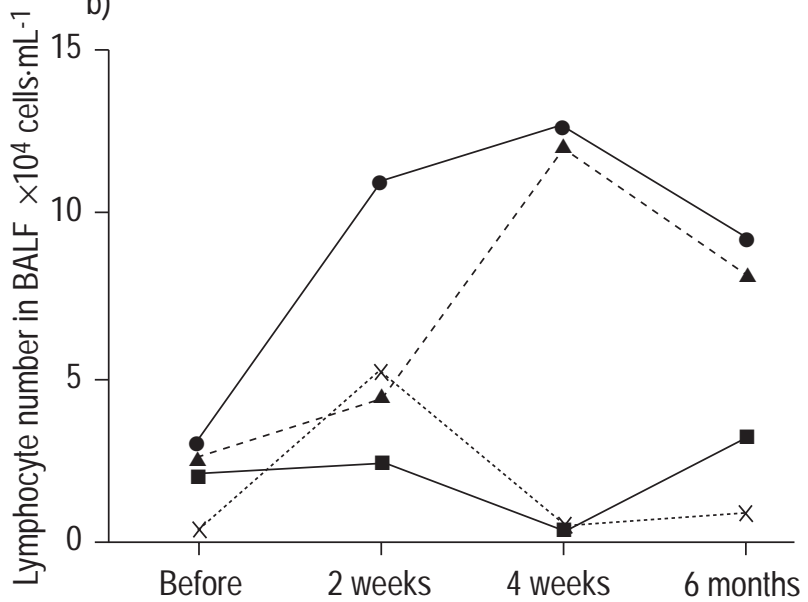

Fig. 3. - Serial changes in the number of a) CD4+ and b) CD8+ lymphocytes in bronchoalveolar lavage fluid (BALF). Values were expressed as the absolute number per millilitre recovered before and after treatment. a) @: CD4+; $\mathbf{0}$ : CD4 + Human leukocyte antigen (HLA)$\mathrm{DR}+; \mathrm{X}: \mathrm{CD} 4+\mathrm{CD} 25+; \mathbf{\Delta}: \mathrm{CD} 4+\mathrm{CD} 45 \mathrm{RO}+. \mathrm{b}) \bullet: \mathrm{CD} 8+; \mathbf{\square}$ CD8 + HLA-DR +; $\mathbf{\Delta}$ : CD8 + CD11b-; X: CD8+ CD11b+.

indicate that the presence of activated CD4+ cells in the lung in AEP may play an important role in eosinophil accumulation through IL-5 production.

Another interesting finding in this case was the persistence of eosinophil accumulation in the lung over several weeks after treatment, although a rapid decrease was noted in the number of activated CD4 lymphocytes and IL5 concentration in BALF. More interestingly, eosinophil count and percentage in BALF returned to normal without additional therapy, and there was no evidence of recurrent disease. In vitro, it has been shown that IL-5 can prolong eosinophil survival for several weeks [13]. One explanation for the persistent accumulation of eosinophils in the alveolar space is the IL-5-induced prolongation of survival of eosinophils in BALF in the early phase of this disease. However, since steroids have a direct effect on eosinophil survival by inducing apoptosis of these cells [13], longterm steroid therapy might lead to an earlier reduction of eosinophils in BALF.

The rapid reduction of activated CD4 lymphocytes and fall of IL-5 concentrations in BALF after therapy suggest that methylprednisolone therapy for 3 days is effective in 
controlling this disease, regardless of eosinophil accumulation. Several studies have suggested a role for IL-5 in the initiation of eosinophilic inflammation. CORRIGAN et al. [14] reported a reduction in the percentage of PB CD4 lymphocytes expressing IL-5 messenger ribonucleic acid (mRNA) and in the secretion of IL-5 protein in asthmatics after corticosteroid therapy. Thus, our results indicate that continuous activation of CD4 lymphocytes and IL-5 production may play a key role in the pathogenesis of AEP, and the effectiveness of corticosteroids may be explained by an inhibition of activated CD4 lymphocyte and cytokine production.

In this patient, the number of $\mathrm{CD} 8+\mathrm{CD} 11 \mathrm{~b}-$ and $\mathrm{CD} 8+$ $\mathrm{CD} 11 \mathrm{~b}+$ cells was not elevated before therapy. The number of CD8+CD11b- cells and CD8+CD11b+ cells in BALF increased 2 weeks after steroid therapy. The number of CD8+CD11b- cells subsequently increased while the number of CD8+CD11b+ cells decreased. Myou et al. [15] reported that $\mathrm{CD} 8+\mathrm{CD} 11 \mathrm{~b}$ - cells in BALF remained elevated during the convalescent phase of measles viral pneumonia. This was probably due to the persistent viral antigen acting as a chronic stimulus in the lung. However, there was no evidence for chronic viral antigen stimulation in this case, that may lead to a high number of CD8+ CD11b-cells during the convalescent phase of this disease. CD8 lymphocytes do not secrete eosinophil-active cytokines, such as IL-3, IL-5 and granulocyte-macrophage colony-stimulating factor (GM-CSF) [13]. Furthermore, a subpopulation of CD8 T-cells exerts a suppressive effect on cell activity. The CD8+ CD11b- phenotype is a suppressor/cytotoxic T-cell subset that suppresses the T-cell proliferative response [16]. In this case, increased CD8+ CD11b- cells in BALF may influence the clearence of eosinophils from the lung and improve the disease state through inhibition of CD4 cell proliferation, reflected by the reduced number of CD4+ CD45RO+ memory cells.

In conclusion, we have described for the first time the possible contribution of activated CD4 lymphocytes and interleukin-5 in the development of acute eosinophilic pneumonia by consecutive analysis of bronchoalveolar lavage fluid and peripheral blood. However, we were unable to establish a direct relationship between activated CD4 cells or interleukin-5 and persistent eosinophilia in bronchoalveolar lavage fluid. Further studies are necessary to establish their role in the pathogenesis of acute eosinophilic pneumonia.

\section{References}

1. Allen JN, Pacht ER, Gadek JE, Davis WB. Acute eosinophilic pneumonia as a reversible cause of non-infectious respiratory failure. $N$ Engl J Med 1989; 321: 569-574.

2. Buchheit J, Eid N, Rodgers G, Feger T, Yakoub O. Acute eosinophilic pneumonia with respiratory failure: a new syndrome? Am Rev Respir Dis 1992; 145: 716-718.
3. Imokawa S, Sato A, Hayakawa H, Toyoshima M, Taniguchi M, Chida K. Possible involvement of an environmental agent in the development of acute eosinophilic pneumonia. Ann Allergy Asthma Immunol 1996; 76: 419422.

4. Miyazaki E, Sugisaki K, Shigenaga T, et al. A case of acute eosinophilic pneumonia caused by inhalation of Trichosporon terrestre. Am J Respir Crit Care Med 1995; 151: 541-543.

5. Okubo Y, Hossain M, Kai R, et al. Adhesion molecules on eosinophils in acute eosinophilic pneumonia. $\mathrm{Am} \mathrm{J}$ Respir Crit Care Med 1995; 151: 1259-1262.

6. Yamaguchi S, Okubo Y, Hossain M, et al. IL-5 pre-dominant in bronchoalveolar lavage fluid and peripheral blood in a patient with acute eosinophilic pneumonia. Intern Med 1995; 34: 65-68.

7. Lopez AF, Sanderson CJ, Gamble JR, Campbell HD, Young IG, Vadas MA. Recombinant human interleukin 5 is a selective activator of human eosinophil chemoattractant. $J$ Exp Med 1988; 167: 219-224.

8. Wang JM, Rambaldi A, Biondi A, Chen ZG, Sanderson CJ, Mantovani A. Recombinant human interleukin 5 is a selective eosinophil chemoattractant. Eur J Immunol 1989; 19: 701-705.

9. Allen JN, Liao Z, Wewers MD, Altenberger EA, Moore SA, Allen ED. Detection of IL-5 and IL-1 receptor antagonists in bronchoalveolar lavage fluid in acute eosinophilic pneumonia. J Allergy Clin Immunol 1996; 97: 1366-1374.

10. Cantrell DA, Smith KA. Transient expression of interleukin 2 receptors. J Exp Med 1983; 158: 1895-1911.

11. Walker C, Bauer W, Braun RK, et al. Activated T cells and cytokines in bronchoalveolar lavages from patients with various lung disease associated with eosinophilia. Am J Respir Crit Care Med 1994; 150: 1038-1048.

12. Okudaira H, Nagami M, Matsuzaki G, et al. T-celldependent accumulation of eosinophils in the lung and its inhibition by monoclonal anti-interleukin-5. Int Arch Allergy Appl Immunol 1991; 94: 171-173.

13. Schleimer RP, Bochner BS. The effects of glucocorticoids on human eosinophils. J Allergy Clin Immunol 1994; 94: 1202-1213.

14. Corrigan CJ, Hamid Q, North J, et al. Peripheral blood CD4 but not CD8 T-lymphocytes in patients with exacerbation of asthma transcribe and translate messenger RNA encoding cytokines which prolong eosinophil survival in the context of a Th2-type pattern: effect of glucocorticoid therapy. Am J Respir Cell Mol Biol 1995; 12: $567-578$.

15. Myou S, Fujimura M, Yasui M, Ueno T, Matsuda T. Bronchoalveolar lavage cell analysis in measles viral pneumonia. Eur Respir J 1993; 6: 1437-1442.

16. Clement L, Dagg M, Landy A. Characterization of human lymphocyte subpopulations: alloreactive cytoxic T-lymphocyte precursor and effector cells are phenotypically distinct from Leu2+ suppressor cells. J Clin Immunol 1984; 4: 395-402. 